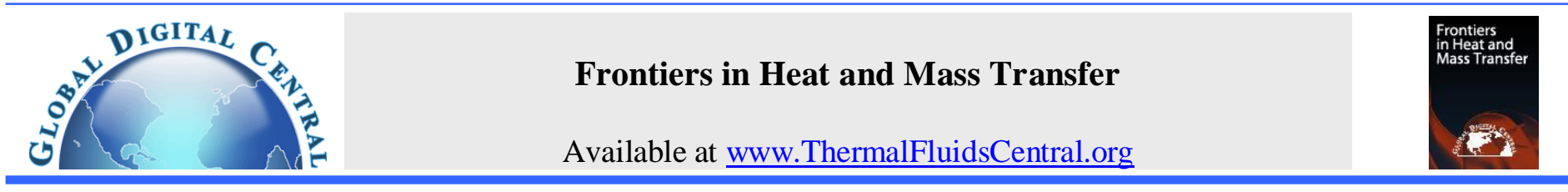

\title{
2D FLOW OF CASSON FLUID WITH NON-UNIFORM HEAT SOURCE/SINK AND JOULE HEATING
}

\author{
Emran Khoshrouye Ghiasi, Reza Saleh* \\ Department of Mechanical Engineering, College of Engineering, Mashhad Branch, Islamic Azad University, Mashhad, Iran
}

\begin{abstract}
In this paper, two-dimensional magnetohydrodynamic (MHD) flow of Casson fluid over a fixed plate under non-uniform heat source/sink and Joule heating is analyzed by the homotopy analysis method (HAM). The governing boundary-layer equations have been reduced to the ordinary differential equations (ODEs) through the similarity variables. The current HAM-series solution is compared and successfully validated by the previous studies. Furthermore, the effects of thermo-physical parameters on the current solution are precisely examined. It is found that the skin friction coefficient and local Nusselt number are greatly affected by the Hartmann number. It can be concluded that employing the Casson fluid together with the suction effect can minimize the rate of heat and mass transfer.
\end{abstract}

Keywords: Nanoparticle, Casson fluid, Heat source/sink, Lorentz force, HAM.

\section{INTRODUCTION}

In general, fluid mechanics can be categorized into two main types: hydraulics and hydrodynamics which are developed through the experimental and theoretical analyses, respectively (Falkner and Skan, 1931). In recent decades, there have been many research studies concerning the hydrodynamics as well as heat and mass transfer theory. In this way, Khan and Azam (2017) investigated unsteady flow of Carreau fluid over a permeable stretching wall with the Lorentz force and suction/injection effect. They solved the governing boundary-layer equations through the bvp $4 c$ function in MATLAB and found that the skin friction coefficient increases with an increase in the Weissenberg number. They also showed that the nanoparticle concentration boundarylayer thickness is significantly affected by the Lewis number. Borrelli et al. (2017) presented a model dealt with the Oberbeck-Boussinesq approximation for three-dimensional (3D) stagnation-point flow of Newtonian fluids. They found that the skin friction coefficient increases with an increase in the Hartmann number. They also illustrated that the reversed flow without the effect of buoyancy force occurs at the minimum value of Hartmann number (i.e., 0.7583). They finally proved that their findings are fully consistent with those of Ramachandran et al. (1988) and Ishak et al. (2008). Rahman et al. (2014) investigated forcedconvection flow of fluids over an exponentially permeable shrinking/stretching wall based on the Buongiorno mathematical model in which the effect of thermophoresis and Brownian motion had been taken into account. They could develop those of Kuznetsov and Nield (2013) and found that the momentum boundary-layer thickness decreases with an increase in the second order slip parameter. Ranjit and Shit (2017) analytically examined the combined effects of Joule heating and zeta potential on the flow past a peristaltically induced microchannel which was supported by those of Tripathi (2013). They also employed the Debye-Hückel approximation technique and found that although the viscous dissipation increases with a decrease in the Brinkman number, the local Nusselt number is a decreasing function of this number.
Besthapu et al. (2017) numerically studied the MHD mixed-convection flow of stratified nanofluids using the finite difference method (FDM). They showed that the nanoparticle concentration boundary-layer thickness increases with an increase in the resistive Lorentz force. Furthermore, they found that the effect of thermal stratification parameter can be neglected at the surface. Sheikholeslami and Ganji (2017) applied the Koo-Kleinstreuer-Li (KKL) model to investigate the MHD flow of $\mathrm{CuO}-\mathrm{H}_{2} \mathrm{O}$ nanofluid over a permeable annulus. They formulated the averaged Nusselt number in terms of inclination angle, Hartmann and Rayleigh numbers, and showed a consistency with those of Khanafer et al. (2003). It is to be noted that mode details are set out in Hedayati and Domairry, 2015; Khoshrouye Ghiasi and Saleh, 2018a, 2018 b, 2017.

As discussed above, analytical and numerical models play a leading role in solving boundary-layer differential equations. This paper focuses on how the HAM (Liao, 1992, 2003) may be implemented to give a solution for MHD flow of Casson fluid combined with the non-uniform heat source/sink, inclined Lorentz force and Joule heating based on the Buongiorno mathematical model (Buongiorno, 2006). It is found that the current findings are in agreement with those of previous studies. In addition, some tables and graphs are provided to signify the effects of thermo-physical parameters on the current solution. To the best of author's knowledge, there have been no reports of this problem being solved to date.

\section{GOVERNING EQUATIONS}

In rheology, one of the well-known non-Newtonian models is the Casson fluid which is defined by the following constitutive equation for an isotropic incompressible flow (Casson, 1959):

$\tau_{i j}= \begin{cases}2\left(\mu_{B}+\frac{p_{y}}{\sqrt{2 \pi}}\right) e_{i j}, & \pi>\pi_{c} \\ 2\left(\mu_{B}+\frac{p_{y}}{\sqrt{2 \pi_{c}}}\right) e_{i j}, & \pi<\pi_{c}\end{cases}$ 
where $\tau_{i j}$ is the shear stress tensor, $\mu_{B}$ is the plastic dynamic viscosity of the fluid, $p_{y}$ is the yield stress, $e_{i j}$ is the $(i, j)$ th component(s) of the strain rate, $\pi\left(=e_{i j} e_{i j}\right)$ is the product of strain rate component(s) and $\pi_{c}$ is the critical value of $\pi$.

For the 2D flow in the Cartesian coordinate system, the velocity, temperature and nanoparticle concentration fields can be expressed as follows:

$\mathbb{V}=[u(x, y), v(x, y)], \mathbb{T}=T(x, y), \mathbb{C}=C(x, y)$,

where $u$ and $v$ are the velocity components along $x$ and $y$ directions, respectively, $T$ is the temperature and $C$ is the nanoparticle concentration.

Utilizing the aforementioned assumptions, the governing continuity, momentum, energy and nanoparticle concentration equations yield:

$\left\{\begin{array}{l}\frac{\partial u}{\partial x}+\frac{\partial v}{\partial y}=0, \\ u \frac{\partial u}{\partial x}+v \frac{\partial u}{\partial y}=v\left(1+\frac{1}{\beta}\right) \frac{\partial^{2} u}{\partial y^{2}}-\frac{\sigma B_{0}^{2}}{\rho} \sin ^{2} \omega u, \\ u \frac{\partial T}{\partial x}+v \frac{\partial T}{\partial y}=\alpha \frac{\partial^{2} T}{\partial y^{2}}+\gamma\left[D_{B} \frac{\partial C}{\partial y} \frac{\partial T}{\partial y}+\frac{D_{T}}{T_{\infty}}\left(\frac{\partial T}{\partial y}\right)^{2}\right] \\ \quad+\frac{1}{\rho C_{p}}\left[q_{n}+\sigma B_{0}^{2} u^{2}\right], \\ u \frac{\partial C}{\partial x}+v \frac{\partial C}{\partial y}=D_{B} \frac{\partial^{2} C}{\partial y^{2}}+\frac{D_{T}}{T_{\infty}} \frac{\partial^{2} T}{\partial y^{2}},\end{array}\right.$

where $v$ is the kinematic viscosity, $\beta$ is the Casson fluid parameter, $\sigma$ is the electrical conductivity, $B_{0}$ is the magnetic field strength, $\rho$ is the density, $\omega$ is the inclination angle of magnetic field, $\alpha$ is the thermal diffusivity, $\gamma\left(=\frac{(\rho C)_{p}}{(\rho C)_{f}}\right)$ is the ratio of effective heat capacity of the nanoparticle to effective heat capacity of the base fluid, $D_{B}$ is the Brownian diffusion coefficient, $D_{T}$ is the thermophoresis diffusion coefficient, $T_{\infty}$ is the ambient temperature, $C_{p}$ is the specific heat at constant pressure and $q_{n}$ is the non-uniform heat source/sink. The associated boundary conditions are given by:

$\left\{\begin{array}{l}\text { at } y=0: u=0, v=v_{w}(x), T=T_{w}, C=C_{w}, \\ \lim _{y \rightarrow \infty} u \rightarrow U_{\infty}, \lim _{y \rightarrow \infty} T \rightarrow T_{\infty}, \lim _{y \rightarrow \infty} C \rightarrow C_{\infty},\end{array}\right.$

where $v_{w}(x)$ is the rate of mass transfer, $T_{w}$ is the wall temperature, $C_{w}$ is the nanoparticle concentration at the wall, $U_{\infty}$ is the free stream velocity and $C_{\infty}$ is the ambient nanoparticle concentration.

In order to derive the similarity solution of equation (3), the following variables can be outlined:

$\phi=\sqrt{U_{0} v} x f(\eta), \eta=y \sqrt{\frac{U_{0}}{v x}}, \theta=\frac{T-T_{\infty}}{T_{w}-T_{\infty}}, \psi=\frac{C-C_{\infty}}{C_{w}-C_{\infty}}$

where $\phi$ is the stream function which is governed by the continuity equation (i.e., $u=\frac{\partial \phi}{\partial y}$ and $v=-\frac{\partial \phi}{\partial x}$ ), $f$ is the similarity function, $\eta$ is the similarity parameter, $\theta$ is the non-dimensional temperature and $\psi$ is the non-dimensional nanoparticle concentration.

The non-uniform heat source/sink involved in equation (3) is given by (Abo-Eldahab and Aziz, 2004; Nandeppanavar et al., 2010; Subhas et al., 2007):

$q_{n}=\frac{k u}{x v}\left[A\left(T_{w}-T_{\infty}\right) \frac{\partial f}{\partial \eta}+B\left(T-T_{\infty}\right)\right]$,

where $A$ and $B$ are the coefficients of space and temperature-dependent heat source/sink, respectively.

By substituting equation (5) into equation (3), the following system of equations can be stated as:

$$
\left\{\begin{array}{c}
\left(1+\frac{1}{\beta}\right) \frac{\partial^{3} f}{\partial \eta^{3}}+f \frac{\partial^{2} f}{\partial \eta^{2}}-\frac{\partial f}{\partial \eta}\left(\frac{\partial f}{\partial \eta}+H a^{2} \sin ^{2} \omega\right)=0, \\
\operatorname{Pr}^{-1} \frac{\partial^{2} \theta}{\partial \eta^{2}}+\frac{1}{2} f \frac{\partial \theta}{\partial \eta}+N b\left(\frac{\partial \theta}{\partial \eta}\right)\left(\frac{\partial \psi}{\partial \eta}\right)+N t\left(\frac{\partial \theta}{\partial \eta}\right)^{2} \\
+P r^{-1}\left(A \frac{\partial f}{\partial \eta}+B \theta\right)+H a^{2} E c\left(\frac{\partial f}{\partial \eta}\right)^{2}=0, \\
\frac{\partial^{2} \psi}{\partial \eta^{2}}+\frac{1}{2} \operatorname{Lef} \frac{\partial \psi}{\partial \eta}+\frac{N t}{N b} \frac{\partial^{2} \theta}{\partial \eta^{2}}=0,
\end{array}\right.
$$

along with the boundary conditions:

$\left\{\begin{array}{l}\text { at } \eta=0: f=S, \frac{\partial f}{\partial \eta}=0, \theta=1, \psi=1, \\ \lim _{\eta \rightarrow \infty} \frac{\partial f}{\partial \eta} \rightarrow 1, \lim _{\eta \rightarrow \infty} \theta \rightarrow 0, \lim _{\eta \rightarrow \infty} \psi \rightarrow 0,\end{array}\right.$

where $H a^{2}=\frac{2 \sigma B_{0}^{2} L}{\rho U_{0}}$ is the square of Hartmann number, $\operatorname{Pr}=\frac{v}{\alpha}$ is the Prandtl number, $N b=\frac{\gamma D_{B}}{v}\left(C_{w}-C_{\infty}\right)$ is the Brownian motion parameter, $N t=\frac{\gamma D_{T}}{v T_{\infty}}\left(T_{w}-T_{\infty}\right)$ is the thermophoresis parameter, $E c=$ $\frac{U_{\infty}^{2}}{C_{p}\left(T_{w}-T_{\infty}\right)}$ is the Eckert number, $L e=\frac{v}{D_{B}}$ is the Lewis number and $S$ is the mass suction parameter. It should be emphasized that the underlined term on the left-hand side of equation (7) indicates the effect of magnetic entropy generation.

The physical quantities of interest are the skin friction coefficient, local Nusselt number and local Sherwood number which can be defined as follows:

$C_{f}=2 \frac{\tau_{w}}{\rho U_{\infty}^{2}}, N u_{x}=\frac{x q_{w}}{k\left(T_{w}-T_{\infty}\right)}, S h_{x}=\frac{x q_{m}}{D_{B}\left(C_{w}-C_{\infty}\right)}$,

where,

$\left\{\begin{array}{l}\tau_{w}=\mu\left(1+\frac{1}{\lambda}\right)\left(\frac{\partial u}{\partial y}\right)_{y=0} \\ q_{w}=-k\left(\frac{\partial T}{\partial y}\right)_{y=0}, \\ q_{m}=-D_{B}\left(\frac{\partial C}{\partial y}\right)_{y=0} .\end{array}\right.$

Substituting equations (5) and (10) into equation (9) yields:

$\left\{\begin{array}{l}C_{f} R e_{x}^{\frac{1}{2}}=\left(1+\frac{1}{\lambda}\right)\left(\frac{\partial^{2} f}{\partial \eta^{2}}\right)_{\eta=0}, \\ N u_{x} / R e_{x}^{\frac{1}{2}}=-\left(\frac{\partial \theta}{\partial \eta}\right)_{\eta=0}, \\ S h_{x} / R e_{x}^{\frac{1}{2}}=-\left(\frac{\partial \phi}{\partial \eta}\right)_{\eta=0},\end{array}\right.$

where $R e_{x}=\frac{x U_{\infty}}{v}$ is the local Reynolds number.

\section{SOLUTION METHOD}

This section provides an overview of the HAM for finding analytical solution of equation (7) together with the boundary conditions given in equation (8). To this end, the initial guesses and auxiliary linear operators can be chosen as follows:

$\left\{\begin{array}{l}f_{0}=S-1+\eta+e^{-\eta}, \theta_{0}=e^{-\eta}, \psi_{0}=e^{-\eta}, \\ L_{f}=\frac{\partial^{3} f}{\partial \eta^{3}}-\frac{\partial f}{\partial \eta}, L_{\theta}=\frac{\partial^{2} \theta}{\partial \eta^{2}}-\theta, L_{\psi}=\frac{\partial^{2} \psi}{\partial \eta^{2}}-\psi,\end{array}\right.$

which have the following properties: 
$\left\{\begin{array}{l}L_{f}\left[C_{1}+C_{2} e^{\eta}+C_{3} e^{-\eta}\right]=0, \\ L_{\theta}\left[C_{4} e^{\eta}+C_{5} e^{-\eta}\right]=0 \\ L_{\psi}\left[C_{6} e^{\eta}+C_{7} e^{-\eta}\right]=0\end{array}\right.$

where $C_{1}, C_{2}, \ldots, C_{7}$ are the arbitrary constants.

Using Liao's theorem (1992), the following zeroth-order deformation equations can be constructed:

$$
\left\{\begin{array}{l}
(1-p) L_{f}\left[f(\eta, p)-f_{0}(\eta)\right]=p h_{f} N_{f}[f(\eta, p)] \\
\quad(1-p) L_{\theta}\left[\theta(\eta, p)-\theta_{0}(\eta)\right] \\
\quad=p h_{\theta} N_{\theta}[f(\eta, p), \theta(\eta, p), \psi(\eta, p)] \\
(1-p) L_{\psi}\left[\psi(\eta, p)-\psi_{0}(\eta)\right] \\
\quad=p h_{\psi} N_{\psi}[f(\eta, p), \theta(\eta, p), \psi(\eta, p)]
\end{array}\right.
$$

in which,

$$
\left\{\begin{array}{l}
N_{f}[f(\eta, p)]=\left(1+\frac{1}{\beta}\right) \frac{\partial^{3} f(\eta, p)}{\partial \eta^{3}}+f(\eta, p) \frac{\partial^{2} f(\eta, p)}{\partial \eta^{2}} \\
\quad-\frac{\partial f(\eta, p)}{\partial \eta}\left(\frac{\partial f(\eta, p)}{\partial \eta}+H a^{2} \sin ^{2} \omega\right), \\
N_{\theta}[f(\eta, p), \theta(\eta, p), \psi(\eta, p)]=\operatorname{Pr}^{-1} \frac{\partial^{2} \theta(\eta, p)}{\partial \eta^{2}}+\frac{1}{2} f(\eta, p) \\
\quad \times \frac{\partial \theta(\eta, p)}{\partial \eta}+N b\left(\frac{\partial \theta(\eta, p)}{\partial \eta}\right)\left(\frac{\partial \psi(\eta, p)}{\partial \eta}\right)+N t\left(\frac{\partial \theta(\eta, p)}{\partial \eta}\right)^{2} \\
\quad r^{-1}\left(A \frac{\partial f(\eta, p)}{\partial \eta}+B \theta(\eta, p)\right)+H a^{2} E c\left(\frac{\partial f(\eta, p)}{\partial \eta}\right)^{2} \\
N_{\psi}[f(\eta, p), \theta(\eta, p), \psi(\eta, p)]=\frac{\partial^{2} \psi(\eta, p)}{\partial \eta^{2}} \\
\quad+\frac{1}{2} \operatorname{Lef}(\eta, p) \frac{\partial \psi(\eta, p)}{\partial \eta}+\frac{N t}{N b} \frac{\partial^{2} \theta(\eta, p)}{\partial \eta^{2}},
\end{array}\right.
$$

with the boundary conditions:

$$
\left\{\begin{array}{l}
\text { at } \eta=0: f(\eta, p)=S, \frac{\partial f(\eta, p)}{\partial \eta}=0, \theta(\eta, p)=1, \\
\psi(\eta, p)=1, \\
\lim _{\eta \rightarrow \infty} \frac{\partial f(\eta, p)}{\partial \eta} \rightarrow 1, \lim _{\eta \rightarrow \infty} \theta(\eta, p) \rightarrow 0, \lim _{\eta \rightarrow \infty} \psi(\eta, p) \rightarrow 0,
\end{array}\right.
$$

where $0 \leq p \leq 1$ is an embedding parameter, $h_{f}, h_{\theta}$ and $h_{\psi}$ are the auxiliary parameters, and $N_{f}, N_{\theta}$ and $N_{\psi}$ are the nonlinear operators.

For $p=0$ and $p=1$, equation (14) is assumed to be:

$$
\left\{\begin{array}{l}
L_{f}\left[f(\eta, 0)-f_{0}(\eta)\right]=0, N_{f}[f(\eta, 1)]=0, \\
L_{\theta}\left[\theta(\eta, 0)-\theta_{0}(\eta)\right]=0, \\
N_{\theta}[f(\eta, 1), \theta(\eta, 1), \psi(\eta, 1)]=0, \\
L_{\psi}\left[\psi(\eta, 0)-\psi_{0}(\eta)\right]=0, \\
N_{\psi}[f(\eta, 1), \theta(\eta, 1), \psi(\eta, 1)]=0 .
\end{array}\right.
$$

Expanding $f, \theta$ and $\psi$ into the Taylor series with respect to $p$ gives:

$$
\left\{\begin{array}{l}
f(\eta, p)=f_{0}(\eta)+\sum_{n=1}^{\infty} f(\eta) p^{n}, \\
\theta(\eta, p)=\theta_{0}(\eta)+\sum_{n=1}^{\infty} \theta(\eta) p^{n}, \\
\psi(\eta, p)=\psi_{0}(\eta)+\sum_{n=1}^{\infty} \psi(\eta) p^{n},
\end{array}\right.
$$

in which,

$$
\begin{aligned}
f(\eta, p) & =\left(\frac{1}{n !} \frac{\partial^{n} f(\eta, p)}{\partial \eta^{n}}\right)_{p=0}, \\
\theta(\eta, p) & =\left(\frac{1}{n !} \frac{\partial^{n} \theta(\eta, p)}{\partial \eta^{n}}\right)_{p=0}, \\
\psi(\eta, p) & =\left(\frac{1}{n !} \frac{\partial^{n} \psi(\eta, p)}{\partial \eta^{n}}\right)_{p=0} .
\end{aligned}
$$

If the initial guesses, auxiliary linear operators and auxiliary parameters are properly chosen, one would get at $p=1$ :

$\left\{\begin{array}{l}f(\eta, p)=\sum_{n=0}^{\infty} f(\eta) \\ \theta(\eta, p)=\sum_{n=0}^{\infty} \theta(\eta) \\ \psi(\eta, p)=\sum_{n=0}^{\infty} \psi(\eta)\end{array}\right.$

Differentiating equation (14) $n$ times with respect to $p$, setting $p=$ 0 and dividing them by $n$ ! gives the following $n$ th-order deformation equations:

$\left\{\begin{array}{l}L_{f}\left[f_{n}(\eta)-\chi_{n} f_{n-1}(\eta)\right]=h_{f} R_{f, n}(\eta), \\ L_{\theta}\left[\theta_{n}(\eta)-\chi_{n} \theta_{n-1}(\eta)\right]=h_{\theta} R_{\theta, n}(\eta), \\ L_{\psi}\left[\psi_{n}(\eta)-\chi_{n} \psi_{n-1}(\eta)\right]=h_{\psi} R_{\psi, n}(\eta),\end{array}\right.$

in which,

$\chi_{n}= \begin{cases}0, & n \leq 1, \\ 1, & n>1,\end{cases}$

$\left\{\begin{array}{l}R_{f, n}(\eta)=\left(1+\frac{1}{\beta}\right) \frac{\partial^{3} f_{n-1}}{\partial \eta^{3}}+\sum_{m=0}^{n-1} f_{m} \frac{\partial^{2} f_{n-m-1}}{\partial \eta^{2}} \\ \quad-\sum_{m=0}^{n-1} \frac{\partial f_{m}}{\partial \eta} \frac{\partial f_{n-m-1}}{\partial \eta}-H a^{2} \sin ^{2} \omega \frac{\partial f_{n-1}}{\partial \eta}, \\ R_{\theta, n}(\eta)=P^{-1} \frac{\partial^{2} \theta_{n-1}}{\partial \eta^{2}}+\frac{1}{2} \sum_{m=0}^{n-1} f_{m} \frac{\partial \theta_{n-m-1}}{\partial \eta} \\ \quad+N b \sum_{m=0}^{n-1} \frac{\partial \psi_{n}}{\partial \eta} \frac{\partial \theta_{n-m-1}}{\partial \eta}+\operatorname{Pr}^{-1}\left(A \frac{\partial \theta_{n}}{\partial \eta}+B \theta_{n-1}\right) \\ \quad+H a^{2} E c \sum_{m=0}^{n-1} \frac{\partial f_{m}}{\partial \eta} \frac{\partial f_{n-m-1}}{\partial \eta}, \\ R_{\psi, n}(\eta)=\frac{\partial^{2} \psi_{n-1}}{\partial \eta^{2}}+\frac{1}{2} L e \sum_{m=0}^{n-1} f_{m} \frac{\partial \psi_{n-m-1}}{\partial \eta}+\frac{N t}{N b} \frac{\partial^{2} \theta_{n-1}}{\partial \eta^{2}},\end{array}\right.$

with the boundary conditions:

$\left\{\begin{array}{l}\text { at } \eta=0: f(\eta)=0, \frac{\partial f(\eta)}{\partial \eta}=0, \theta(\eta)=0, \psi(\eta)=0, \\ \lim _{\eta \rightarrow \infty} \frac{\partial f(\eta)}{\partial \eta} \rightarrow 0, \lim _{\eta \rightarrow \infty} \theta(\eta) \rightarrow 0, \lim _{\eta \rightarrow \infty} \psi(\eta) \rightarrow 0 .\end{array}\right.$

The general solutions for equation (21) in terms of particular solutions (i.e., $f^{\star}, \theta^{\star}$ and $\psi^{\star}$ ) can be expressed as follows:

$\left\{\begin{array}{l}f_{n}(\eta)=f_{n}^{\star}(\eta)+C_{1}+C_{2} e^{\eta}+C_{3} e^{-\eta}, \\ \theta_{n}(\eta)=\theta_{n}^{\star}(\eta)+C_{4} e^{\eta}+C_{5} e^{-\eta} \\ \psi_{n}(\eta)=\psi_{n}^{\star}(\eta)+C_{6} e^{\eta}+C_{7} e^{-\eta}\end{array}\right.$

in which,

$\left\{\begin{array}{l}C_{2}=C_{4}=C_{6}=0, C_{1}=-\left(C_{3}+f_{n}^{\star}(0)\right), \\ C_{3}=\frac{\partial f_{n}^{\star}(0)}{\partial \eta}, C_{5}=\theta_{n}^{\star}(0), C_{7}=\psi_{n}^{\star}(0) .\end{array}\right.$

The square residual errors can be defined as (Liao, 2010):

$\left\{\begin{array}{l}\Delta_{f, n}=\frac{1}{i+1} \sum_{j=0}^{i}\left\{N_{f}\left[\sum_{r=0}^{n} f(\eta)\right]_{\eta=j \delta \eta}\right\}^{2}, \\ \Delta_{\theta, n}=\frac{1}{i+1} \\ \quad \times \sum_{j=0}^{i}\left\{N_{\theta}\left[\sum_{r=0}^{n} f(\eta), \sum_{r=0}^{n} \theta(\eta), \sum_{r=0}^{n} \psi(\eta)\right]_{\eta=j \delta \eta}\right\}^{2}, \\ \Delta_{\psi, n}=\frac{1}{i+1} \\ \quad \times \sum_{j=0}^{i}\left\{N_{\psi}\left[\sum_{r=0}^{n} f(\eta), \sum_{r=0}^{n} \theta(\eta), \sum_{r=0}^{n} \psi(\eta)\right]_{\eta=j \delta \eta}\right\}^{2},\end{array}\right.$

where $i=20$ and $\delta \eta=0.5$. 


\section{RESULTS AND DISCUSSION}

This section deals with the previously outlined HAM-series solution for heat and mass transfer analysis in the MHD flow of Casson fluid subjected to inclined Lorentz force and Joule heating. In this way, the comparisons and parametric studies are made to investigate the validity and accuracy of the current solution. In this paper the pertinent parameters, unless stated otherwise, are listed as follows:

$\left\{\beta=0.4, \mathrm{Ha}=1, \omega=45^{\circ}, S=1, \operatorname{Pr}=0.7\right.$,

$\{N b=N t=0.5, A=0.05, B=-0.05, E c=0.1, L e=1.3$.

Table 1 represents the variation of auxiliary parameters and its square residual errors at any order of approximation. From this table, it is seen that the allowable values of auxiliary parameters can be chosen by minimizing the square residual errors. Therefore, the current findings are provided using the optimized 20th-order of approximation (i.e., $h_{f}=$ $-0.8169, h_{\theta}=-0.8246$ and $\left.h_{\psi}=-1.0962\right)$.

Effect of the Hartmann number $\mathrm{Ha}$ on the skin friction coefficient $-C_{f} R e_{x}^{\frac{1}{2}}$ is depicted in Fig. 1 for different values of inclination angle of magnetic field $\omega$. As this figure shows, $-C_{f} R e_{x}^{\frac{1}{2}}$ increases with an increase in $\omega$ which is due to the presence of resistive Lorentz force. It should be noted that the Casson fluid is affected by the viscous force while the Lorentz force tends to decelerate flow of the fluid and retards its motion (Khoshrouye Ghiasi and Saleh, 2018c). Moreover, unlike $\omega=$ $0^{\circ},-C_{f} R e_{x}^{\frac{1}{2}}$ increases with an increase in $\mathrm{Ha}$. Since $\omega=0^{\circ}$ the effect of Hartmann number on the momentum boundary-layer thickness is negligible.

Table 1 selection of auxiliary parameters

\begin{tabular}{|c|c|c|c|c|c|c|}
\hline$n$ & $h_{f}$ & $\Delta_{f, n}$ & $h_{\theta}$ & $\Delta_{\theta, n}$ & $h_{\psi}$ & $\Delta_{\psi, n}$ \\
\hline 2 & -0.7614 & $6.14 \times 10^{-8}$ & -0.8006 & $5.20 \times 10^{-5}$ & -1.0070 & $4.54 \times 10^{-7}$ \\
\hline 4 & -0.7698 & $3.0 .3 \times 10^{-8}$ & -0.8025 & $4.68 \times 10^{-5}$ & -1.0195 & $2.19 \times 10^{-7}$ \\
\hline 6 & -0.7740 & $9.26 \times 10^{-9}$ & -0.8049 & $3.90 \times 10^{-5}$ & -1.0296 & $9.80 \times 10^{-8}$ \\
\hline 8 & -0.7788 & $5.99 \times 10^{-9}$ & -0.8082 & $3.51 \times 10^{-5}$ & -1.0399 & $7.22 \times 10^{-8}$ \\
\hline 10 & -0.7939 & $1.04 \times 10^{-9}$ & -0.8107 & $3.08 \times 10^{-5}$ & -1.0498 & $4.93 \times 10^{-8}$ \\
\hline 12 & -0.7983 & $8.06 \times 10^{-10}$ & -0.8131 & $2.79 \times 10^{-5}$ & -1.0585 & $2.30 \times 10^{-8}$ \\
\hline 14 & -0.8032 & $5.12 \times 10^{-10}$ & -0.8159 & $2.32 \times 10^{-5}$ & -1.0694 & $9.79 \times 10^{-9}$ \\
\hline 16 & -0.8070 & $2.97 \times 10^{-10}$ & -0.8190 & $2.01 \times 10^{-5}$ & -1.0790 & $6.95 \times 10^{-9}$ \\
\hline 18 & -0.8111 & $8.25 \times 10^{-11}$ & -0.8214 & $1.71 \times 10^{-5}$ & -1.0883 & $3.93 \times 10^{-9}$ \\
\hline 20 & -0.8169 & $4.89 \times 10^{-11}$ & -0.8246 & $1.46 \times 10^{-5}$ & -1.0962 & $1.09 \times 10^{-9}$ \\
\hline
\end{tabular}

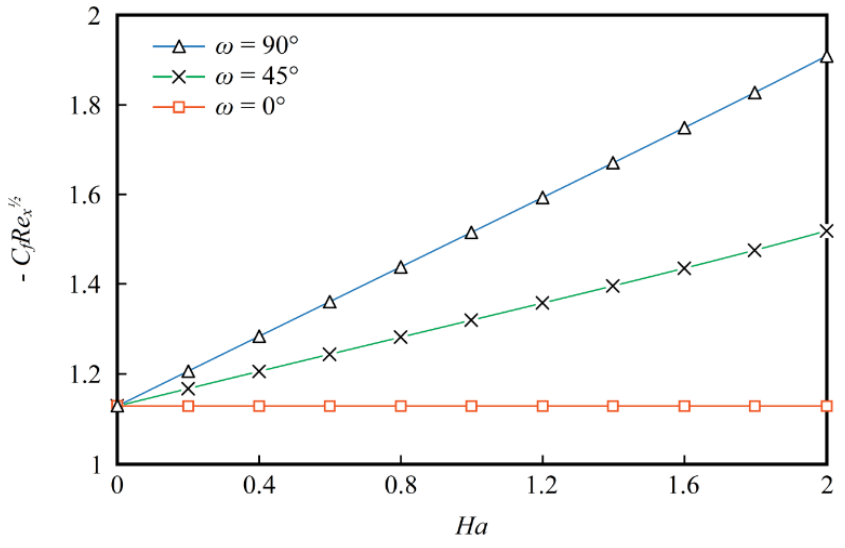

Fig. 1 Variation of $-C_{f} R e_{x}^{\frac{1}{2}}$ versus $H a$ for $\omega=0^{\circ}, \omega=45^{\circ}$ and $\omega=90^{\circ}$
Due to the effect of yield stress $p_{y}$ on the Casson fluid given in equation (1), one can observe from Fig. 2 that the velocity distribution decreases with an increase in the Casson fluid parameter $\beta$. This is because, an increase in $\beta$ leads to a decrease in $p_{y}$, which is replaced by the Newtonian fluid. This fact is also illustrated in Aziz (2016) and Khoshrouye Ghiasi and Saleh (2019). It is worth mentioning that some previous studies (Raju et al., 2017; Raju and Sandeep, 2017) suggest ascending behavior of the velocity distribution with an increase in $\beta$ which is largely due to the domination of buoyancy force.

Table 2 tabulates the effect of Hartmann number $\mathrm{Ha}$ and mass suction parameter $S$ on the values of $\left(\frac{\partial^{2} f}{\partial \eta^{2}}\right)_{\eta=0}$. According to the results reported in this table, it is observed that $\left(\frac{\partial^{2} f}{\partial \eta^{2}}\right)_{\eta=0}$ is an increasing function of $\mathrm{Ha}$ and $S$ simultaneously. It should be noted that for large values of mass suction parameter, the axial velocity decreases.

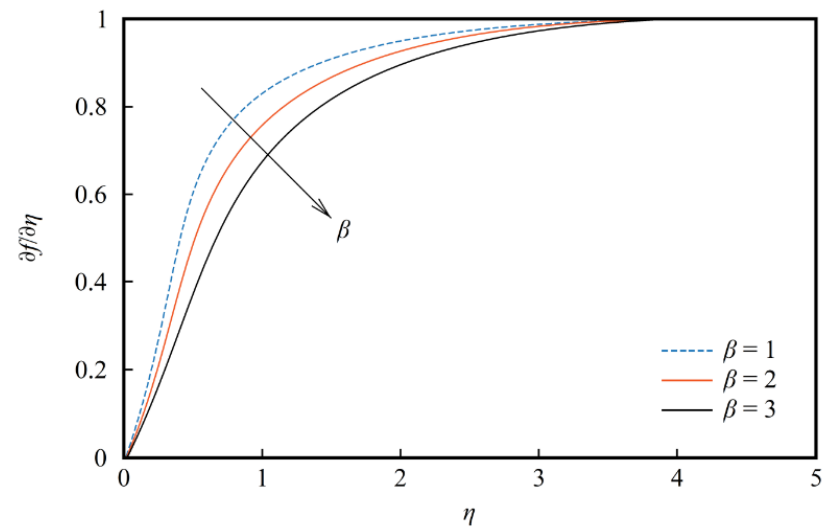

Fig. 2 Variation of $\frac{\partial f}{\partial \eta}$ for $\beta=1, \beta=2$ and $\beta=3$

Table 2 Values of the skin friction coefficient for $\mathrm{Ha}$ and $\mathrm{S}$

\begin{tabular}{|c|c|c|}
\hline$H a$ & $S$ & $\left(\frac{\partial^{2} f}{\partial \eta^{2}}\right)_{\eta=0}$ \\
\hline 1 & & 1.1665 \\
\hline 2 & & 1.2796 \\
\hline 3 & & 1.3481 \\
\hline 4 & & 1.4195 \\
\hline 1 & 0.6 & 0.9228 \\
\hline & 0.7 & 0.9396 \\
\hline & 0.8 & 0.9451 \\
\hline & 0.9 & 0.9517 \\
\hline
\end{tabular}

Table 3 shows a comparison between the current solution and those of Khan and Pop (2010) and Wang (1989) to determine the values of $-\left(\frac{\partial \theta}{\partial \eta}\right)_{\eta=0}$. The results in this table are provided by $\beta \rightarrow \infty, H a=0.1$, $E c=0.1, L e=10$ and $\omega=S=N b=N t=A=B=0$. It is seen from Table 3 that $-\left(\frac{\partial \theta}{\partial \eta}\right)_{\eta=0}$ increases with an increase in $\operatorname{Pr}$. Moreover, the relative error between the current solution and those of Khan and Pop (2010) and Wang (1989) does not exceed $0.047 \%$ and $0.111 \%$, respectively. Hence, the reliability of the current solution is verified.

As mentioned earlier, the irreversibility of the Joule heating process can be measured by the magnetic entropy generation (Bejan, 1982). This fact is illustrated in Fig. 3 for the variation of local Nusselt number $N u_{x} / R e_{x}^{\frac{1}{2}}$ versus $H a$. From this figure, it can be seen that $N u_{x} / R e_{x}^{\frac{1}{2}}$ decreases with an increase in the Eckert number $E c$ which is due to the stored energy in the fluid. This observation can also be considered as an 
optimization criterion for minimizing the entropy generation (Bejan, 1995).

To investigate the effect of non-uniform heat source/sink on the temperature distribution, the variation of $\theta(\eta)$ for different values of $A$ and $B$ is depicted in Fig. 4. As this figure shows, $\theta(\eta)$ increases with an increase in $A$ or $B$ which is due to an increase in the thermal boundarylayer thickness. Furthermore, Fig. 4 emphasizes that the surface temperature $\theta(0)$ increases with an increase in $A$.

Table 3 Values of $-\left(\frac{\partial \theta}{\partial \eta}\right)_{\eta=0}$ compared with those of Khan and Pop (2010) and Wang (1989)

\begin{tabular}{|c|c|c|c|}
\hline$P r$ & Khan and Pop (2010) & Wang (1989) & Current solution \\
\hline 0.07 & 0.0663 & 0.0656 & 0.0661 \\
\hline 0.2 & 0.1691 & 0.1691 & 0.1691 \\
\hline 0.7 & 0.4539 & 0.4539 & 0.4538 \\
\hline 2 & 0.9113 & 0.9114 & 0.9114 \\
\hline 7 & 1.8954 & 1.8954 & 1.8954 \\
\hline 20 & 3.3539 & 3.3539 & 3.3539 \\
\hline 70 & 6.4621 & 6.4622 & 6.4621 \\
\hline
\end{tabular}

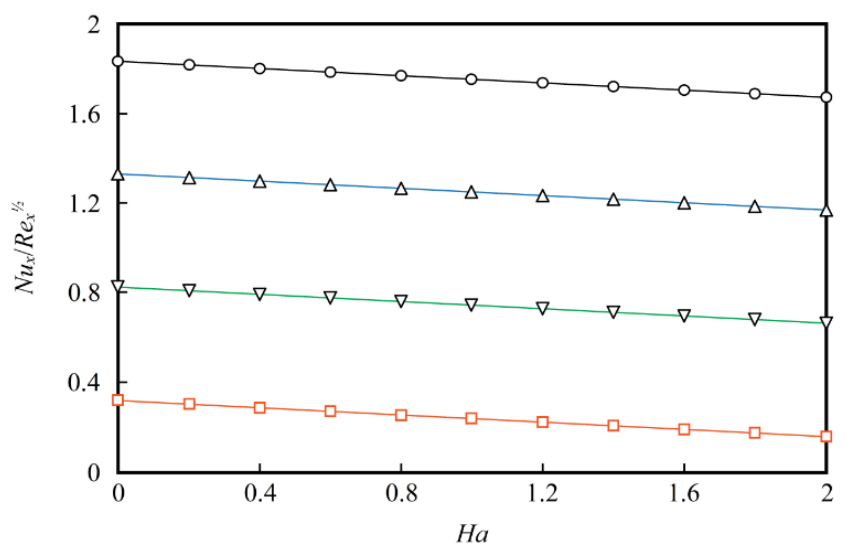

$\multimap-E c=0.1 \rightarrow \triangle E c=0.2 \rightarrow-E c=0.3 \rightarrow \square-E c=0.4$

Fig. 3 Variation of $N u_{x} / R e_{x}^{\frac{1}{2}}$ versus $H a$ for $E c=0.1, E c=0.2$, $E c=0.3$ and $E c=0.4$

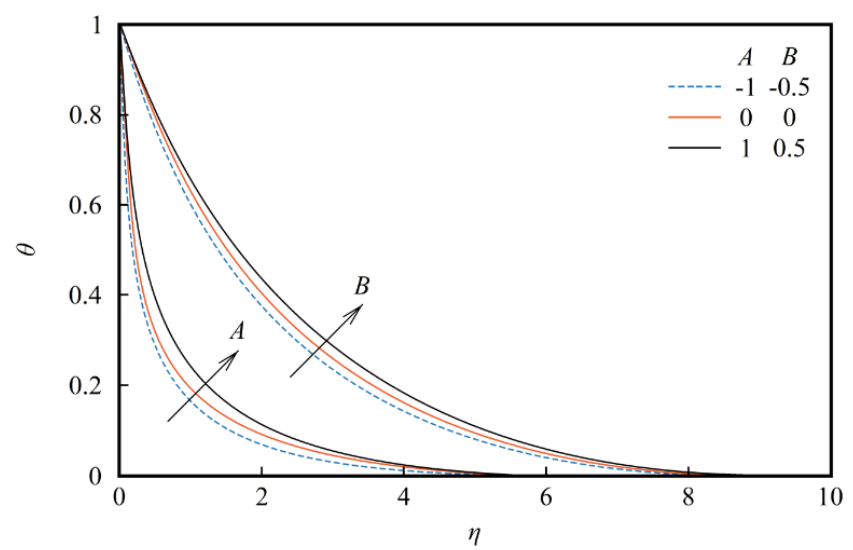

Fig. 4 Variation of $\theta(\eta)$ for $A=-1, A=0$ and $A=1$, and, $B=$ $-0.5, B=0$ and $B=0.5$

Table 4 shows the effect of Brownian motion parameter $\mathrm{Nb}$ and thermophoresis parameter $N t$ on the variation of $-\left(\frac{\partial \theta}{\partial \eta}\right)_{\eta=0}$. It can be observed from this table that $-\left(\frac{\partial \theta}{\partial \eta}\right)_{\eta=0}$ increases with an increase in $N b$, which is due to an interaction between the Brownian motion and thermal conductivity. Also, Table 4 shows that $-\left(\frac{\partial \theta}{\partial \eta}\right)_{\eta=0}$ clearly decreases with an increase in $N t$. Therefore, it is essential to account for the effect Brownian diffusion and thermophoresis in the Buongiorno mathematical model.

A comparison between the current solution and those of Afify and Elgazery (2016) is reported in Table 5 to determine the effect of $N t$ and $N b$ on the variation of $-\left(\frac{\partial \psi}{\partial \eta}\right)_{\eta=0}$. It is noted that the pertinent parameters utilized by Afify and Elgazery (2016) (i.e., $\beta \rightarrow \infty, \operatorname{Pr}=$ $L e=10$ and $H a=\omega=S=A=B=E c=0$ ) agree with those presented in this table. It is found that the relative error between the current solution and those of Afify and Elgazery (2016) equals to $0.331 \%$ in all cases.

Fig. 5 illustrates the variation of nanoparticle concentration $\psi(\eta)$ for different values of Lewis number $L e$. From this figure, it is seen that $\psi(\eta)$ decreases with an increase in $L e$ which is due to the effect of $D_{B}$.

Table 4 Values of the local Nusselt number for $N b$ and $N t$

\begin{tabular}{|c|c|c|}
\hline$N b$ & $N t$ & $-\left(\frac{\partial \theta}{\partial \eta}\right)_{\eta=0}$ \\
\hline 0.1 & 0.5 & 0.5059 \\
\hline 0.2 & & 0.5625 \\
\hline 0.3 & & 0.6148 \\
\hline 0.4 & & 0.6809 \\
\hline 0.1 & 0.6 & 0.4730 \\
\hline & 0.7 & 0.4415 \\
\hline & 0.8 & 0.4120 \\
\hline & 0.9 & 0.4796 \\
\hline
\end{tabular}

Table 5 Values of $-\left(\frac{\partial \psi}{\partial \eta}\right)_{\eta=0}$ compared with those of Afify and Elgazery (2016)

\begin{tabular}{|c|c|c|c|}
\hline$N t$ & $N b$ & Afify and Elgazery (2016) & Current solution \\
\hline 0.1 & 0.1 & 2.2774 & 2.2637 \\
\hline 0.2 & & 2.2490 & 2.2388 \\
\hline 0.3 & & 2.2229 & 2.2181 \\
\hline 0.4 & & 2.1992 & 2.1907 \\
\hline 0.1 & 0.2 & 2.3110 & 2.3089 \\
\hline & 0.3 & 2.3299 & 2.3216 \\
\hline & 0.4 & 2.3458 & 2.3389 \\
\hline & 0.5 & 2.3560 & 2.3501 \\
\hline
\end{tabular}

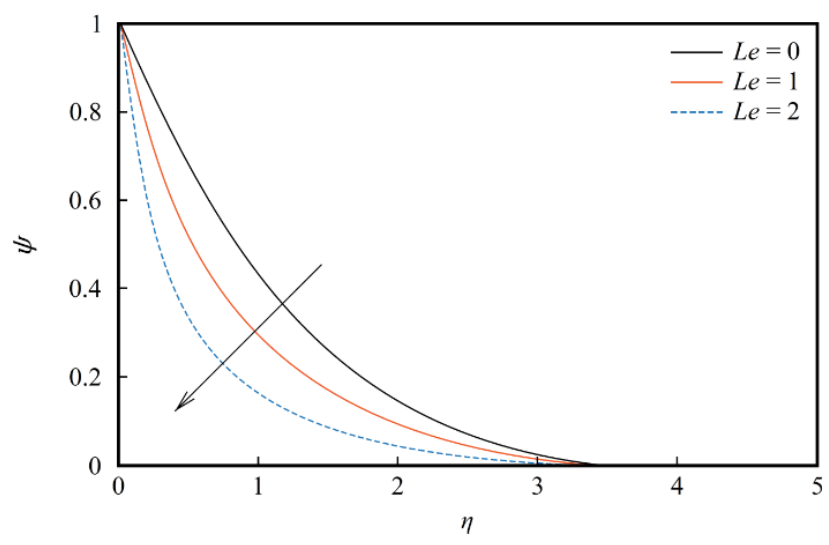

Fig. 5 Variation of $\psi(\eta)$ for $L e=0, L e=1$ and $L e=2$ 


\section{CONCLUSIONS}

This paper aimed to study the effect of non-uniform heat sink/source, Joule heating and inclined Lorentz force on flow of Casson fluid based on the Buongiorno mathematical model. The governing boundary-layer equations correspond to the continuity, momentum, energy and nanoparticle concentration equations are derived, and solved through the HAM. It was found that the rate of heat transfer is affected by the Brownian diffusion and thermophoresis. Moreover, the HAM findings were compared and validated by those previous studies available in the literature. One can also conclude that this paper would be worthwhile to further explore this problem with different boundary conditions, solution methodologies and geometries.

\section{REFERENCES}

Falkner, V.M., Skan, S.W., 1931, "Some approximate solutions of the boundary layer equations," Philosophical Magazine, 12(80), 865-896. http://dx.doi.org/10.1080/14786443109461870

Khan, M., Azam, M., 2017, "Unsteady heat and mass transfer mechanisms in MHD Carreau nanofluid flow," Journal of Molecular Liquids, 225, 554-562.

http://dx.doi.org/10.1016/j.molliq.2016.11.107

Borrelli, G., Giantesio, M.C., Patria, N.C., Roşca, A.V., Pop, I., 2017, "Buoyancy effects on the 3D MHD stagnation-point flow of a Newtonian fluid," Communications in Nonlinear Science and Numerical Simulation, 43, 1-13. http://dx.doi.org/10.1016/j.cnsns.2016.06.022

Ramachandran, N., Chen, T.S., Armaly, B.F., 1988, "Mixed convection in stagnation flows adjacent to vertical surfaces," Journal of Heat Transfer, 110(2), 373-377.

http://dx.doi.org/10.1115/1.3250494

Ishak, A., Nazar, R., Arifin, N.M., Pop, I., 2008, "Dual solutions in mixed convection flow near a stagnation point on a vertical porous plate," International Journal of Thermal Sciences, 47(4), 417-422. http://dx.doi.org/10.1016/j.ijthermalsci.2007.03.005

Rahman, M.M., Roşca, A.V., Pop, I., 2014, "Boundary layer flow of a nanofluid past a permeable exponentially shrinking/stretching surface with second order slip using Buongiorno's model," International Journal of Heat and Mass Transfer, 77, 1133-1143.

http://dx.doi.org/10.1016/j.ijheatmasstransfer.2014.06.013

Kuznetsov, A.V., Nield, D.A., 2013, "The Cheng-Minkowycz problem for natural convective boundary layer flow in a porous medium saturated by a nanofluid: A revised model," International Journal of Heat and Mass Transfer, 65, 682-685.

http://dx.doi.org/10.1016/j.ijheatmasstransfer.2013.06.054

Ranjit, N.K., Shit, G.C., 2017, “Joule heating effects on electromagnetohydrodynamic flow through a peristaltically induced micro-channel with different zeta potential and wall slip," Physica A: Statistical Mechanics and its Applications, 482, 458-476.

http://dx.doi.org/10.1016/j.physa.2017.04.072

Tripathi, D., 2013, "Study of transient peristaltic heat flow through a finite porous channel," Mathematical and Computer Modelling, 57(5-6), 1270-1283.

http://dx.doi.org/10.1016/j.mcm.2012.10.030

Besthapu, P., Ul Haq, R., Bandari, S., Al.Madalla, Q.M., 2017, "Mixed convection flow of thermally stratified MHD nanofluid over an exponentially stretching surface with viscous dissipation effect," Journal of the Taiwan Institute of Chemical Engineers, 71, 307-314.

http://dx.doi.org/10.1016/j.jtice.2016.12.034
Sheikholeslami, M., Ganji, D.D., 2017, "Transportation of MHD nanofluid free convection in a porous semi annulus using numerical approach," Chemical Physics Letters, 669, 202-210.

http://dx.doi.org/10.1016/j.cplett.2016.12.045

Khanafer, K., Vafai, K., Lightstone, M., 2003, "Buoyancy-driven heat transfer enhancement in a two-dimensional enclosure utilizing nanofluids," International Journal of Heat and Mass Transfer, 46(19), 3639-3653.

http://dx.doi.org/10.1016/S0017-9310(03)00156-X

Hedayati, F., Domairry, G., 2015, "Effects of nanoparticle migration and asymmetric heating on mixed convection of $\mathrm{TiO}_{2}-\mathrm{H}_{2} \mathrm{O}$ nanofluid inside a vertical microchannel," Powder Technology, 272, 250-259. http://dx.doi.org/10.1016/j.powtec.2014.12.003

Khoshrouye Ghiasi, E., Saleh, R., 2018a, "Non-dimensional optimization of magnetohydrodynamic Falkner-Skan fluid flow," INAE Letters, 3(3), 143-147.

http://dx.doi.org/10.1007/s41403-018-0043-2

Khoshrouye Ghiasi, E., Saleh, R., 2018b, "Constructing analytic solutions on the Tricomi equation," Open Physics, 16(1), 143-148. http://dx.doi.org/10.1515/phys-2018-0022

Khoshrouye Ghiasi, E., Saleh, R., 2017, “A mathematical approach based on the homotopy analysis method: Application to solve the nonlinear Harry-Dym (HD) equation," Applied Mathematics, 8(11), 1546-1562.

http://dx.doi.org/10.4236/am.2017.811113

Liao, S.J., 2003, Beyond perturbation: Introduction to the homotopy analysis method, Chapman \& Hall/CRC Press, Boca Raton.

Liao, S.J., 1992, The proposed homotopy analysis techniques for the solution of nonlinear problems, PhD. Thesis, Shanghai Jiao Tong University.

Buongiorno, J., 2006, “Convective transport in nanofluids," Journal of Heat Transfer, 128(3), 240-250.

http://dx.doi.org/10.1115/1.2150834

Casson, N., 1959, Rheology of disperse systems, C.C. Mill., London.

Abo-Eldahab, E.M., Aziz, M.A.E., 2004, "Blowing/suction effect on hydromagnetic heat transfer by mixed convection from an inclined continuously stretching surface with internal heat generation/absorption," International Journal of Thermal Sciences, 43(7), 709-719.

http://dx.doi.org/10.1016/j.ijthermalsci.2004.01.005

Nandeppanavar, M.M., Subhas Abel, M., Tawade, J., 2010, "Heat transfer in Walter's liquid B fluid over an impermeable stretching sheet with non-uniform heat source/sink and elastic deformation," Communications in Nonlinear Science and Numerical Simulation, 15(7), 1791-1802.

http://dx.doi.org/10.1016/j.cnsns.2009.07.009

Subhas Abel, M., Siddheshwar, P.G., Nandeppanavar, M.M., 2007, "Heat transfer in a viscoelastic boundary layer flow over a stretching sheet with viscous dissipation and non-uniform heat source," International Journal of Heat and Mass Transfer, 50(5-6), 960-966. http://dx.doi.org/10.1016/j.ijheatmasstransfer.2006.08.010

Liao, S.J., 2010, “An optimal homotopy-analysis approach for strongly nonlinear differential equations," Communications in Nonlinear Science and Numerical Simulation, 15(8), 2003-2016. http://dx.doi.org/10.1016/j.cnsns.2009.09.002 
Khoshrouye Ghiasi, E., Saleh, R., 2018c, "Unsteady shrinking embedded horizontal sheet subjected to inclined Lorentz force and Joule heating, an analytical solution," Results in Physics, 11, 65-71.

http://dx.doi.org/10.1016/j.rinp.2018.07.026

Aziz, M.A.E., 2016, "Effects of variable thermal conductivity with thermal radiation on MHD flow and heat transfer of Casson liquid film over an unsteady stretching surface," Brazilian Journal of Physics, 46(5), 516-525.

http://dx.doi.org/10.1007/s13538-016-0442-3

Khoshrouye Ghiasi, E., Saleh, R., 2019, "Nonlinear stability and thermomechanical analysis of hydromagnetic Falkner-Skan Casson conjugate fluid flow over an angular-geometric surface based on Buongiorno's model using homotopy analysis method and its extension," Pramana, 92(1), 1-12.

http://dx.doi.org/10.1007/s12043-018-1667-1

Raju, C.S.K., Hoque, M.M., Sivasankar, T., 2017, "Radiative flow of Casson fluid over a moving wedge filled with gyrotactic microorganisms," Advanced Powder Technology, 28(2), 575-583. http://dx.doi.org/10.1016/j.apt.2016.10.026
Raju, C.S.K., Sandeep, N., 2017, "MHD slip flow of a dissipative Casson fluid over a moving geometry with heat source/sink: A numerical study," Acta Astronautica, 133, 436-443.

http://dx.doi.org/10.1016/j.actaastro.2016.11.004

Khan, W.A., Pop, I., 2010, "Boundary-layer flow of a nanofluid past a stretching sheet," International Journal of Heat and Mass Transfer, 53(11-12), 2477-2483.

http://dx.doi.org/10.1016/j.ijheatmasstransfer.2010.01.032

Wang, C.Y., 1989, "Free convection on a vertical stretching surface," Journal of Applied Mathematics and Mechanics, 69(11), 418-420. http://dx.doi.org/10.1002/zamm.19890691115

Bejan, A., 1982, Entropy generation through heat and fluid flow, Wiley, New York.

Bejan, A., 1995, Entropy generation minimization, Chapman \& Hall/CRC Press, Boca Raton.

Afify, A.A., Elgazery, N.S., Effect of a chemical reaction on magnetohydrodynamic boundary layer flow of a Maxwell fluid over a stretching sheet with nanoparticles," Particuology, 29, 154-161. http://dx.doi.org/10.1016/j.partic.2016.05.003 